\title{
CORRESPONDENCE
}

\section{Scientists stand by decision to join Mbeki's AIDS panel}

SIR - Your Editorial 'The cost of silence?' (Nature 456, 545; 2008) questions our decision - as scientists who opposed dissident theories - to participate in the now-discredited AIDS advisory panel set up by former South African president Thabo Mbeki.

We had no role in, and did not see or approve, the panel's report (Nature 410, 730; 2001). Each of us had agreed independently to join the panel, guided by our consciences as scientists in a young democracy and without prior knowledge of who else had been invited to participate. To us, it provided an opportunity to present Mbeki with the alternative viewpoint and the compelling scientific evidence that HIV causes AIDS, in the hope that rationality would prevail.

However, Mbeki's antipathy to antiretroviral drugs was influenced by documents from and interactions with AIDS dissidents that predated the setting up of the panel. We underestimated the strength of his dissident views on AIDS and how little impact sound science would eventually make on them.

Sadly, our advice to Mbeki on AIDS causation and antiretroviral treatment was rejected. We cannot, therefore, be numbered among those held accountable for Mbeki's decisions, which led to the loss of many thousands of lives in South Africa through lack of access to antiretroviral therapy.

That we failed to change Mbeki's opinions on AIDS is a matter of record. But your Editorial is unreasonable in implying with the benefit of hindsight - that scientists could have foreseen this failure and therefore should not have signed up to an opportunity to give the president critically important information that might have saved the lives of their fellow-countrymen.

We stand by our decision to participate in the Mbeki panel. We have an obligation to our country, which is suffering the worst AIDS epidemic in the world, to do everything in our power to provide our political decision-makers with the best scientific advice, whether or not they are a priori opposed to or supportive of our views.

Salim S. Abdool Karim, Hoosen M. Coovadia, Malegapuru W. Makgoba CAPRISA, Doris Duke Medical Research Institute, Nelson R. Mandela School of Medicine, University of KwaZulu-Natal, Private Bag X7, Congella 4013, South Africa e-mail:karims1@ukzn.ac.za

\section{When winning a Nobel prize seems to run in the family}

SIR — In his Correspondence 'You're the best man for this job, son. What a coincidence!', Albert Ruggi's suspicions about the process by which the offspring of professors are deemed to be the best candidates for new positions may well be justified (Nature 456, 870; 2008). On the other hand, a few rare families just do produce generations of eminent scientists. For example, there are at least seven parent-child pairs of Nobel laureates.

Four of these were in physics: the Thomsons (J. J. in 1906 and George in 1937), Braggs (William and Lawrence together in 1915), Bohrs (Niels in 1922 and his son Aage in 1975) and Siegbahns (Manne in 1924 and his son Kai in 1981). Marie Curie and her daughter Irène Joliot-Curie both won the Nobel Prize in Chemistry (1911 and 1935), after Marie and her husband, Pierre, had won the physics Nobel in 1903.

The Kornbergs branched out more (Arthur, physiology or medicine, 1959; Roger, chemistry, 2006), as did Hans von EulerChelpin (chemistry, 1929) and his son Ulf von Euler (physiology or medicine, 1970).

Jay M. Pasachoff California Institute of Technology 150-21, Pasadena, California 91125, USA e-mail:jmp@caltech.edu
Ecologists should join astronomers to oppose light pollution

SIR — In his Commentary 'Time to turn off the lights' (Nature 457, 27; 2009), astronomer Malcolm Smith argues for darker skies. Ecologists would also do well to support the International Year of Astronomy, considering the potentially severe impact of light pollution on some biological systems. Moths, for example, as well as the bird migrations Smith mentions, are adversely affected by light pollution.

In most instances, the origin of atmospheric and ecological light pollution is identical. So ecologists should back initiatives stimulated this year by physicists, to help raise awareness of the undesirability of light pollution across different disciplines. Josef Settele UFZ, Helmholtz Centre for Environmental Research, TheodorLieser-Strasse 4, 06120 Halle, Germany e-mail: Josef.Settele@ufz.de

\section{Lindauer's genius showed evolution in a simple experiment}

SIR - I was saddened to learn of the death of Martin Lindauer, an under-appreciated hero of science. Thomas D. Seeley, in his Obituary (Nature 456, 718; 2008), describes experiments from Lindauer's Communication Among Social Bees (Harvard Univ. Press, 1961) that demonstrate his talent. The book also includes experiments that possess what physicist I. I. Rabi used to call 'witz': an unexpected twist that elevates an experiment to a higher level.

Living in an enclosed, sheltered space, the honeybee Apis mellifera performs its communicative dance on a vertical surface in the dark, using gravity as a substitute for the direction of the Sun. By depriving them of a vertical surface and giving them a direct view of the Sun, Lindauer forced them to revert to the more primitive, Sun-directed dance of their dwarf Indian relative, Apis florea.

In closing the gap between a primitive and an advanced condition, Lindauer possibly produced the best-ever experimental evidence for evolution. Scientists concerned with evolution of human language and mind might ponder his success.

William L. Abler Department of Geology, The Field Museum, 1400 South Lake Shore Drive, Chicago, Illinois 60605, USA e-mail: wabler@fieldmuseum.org

\section{Culture clash in Chinese university: a response}

SIR - In your Editoral 'Culture clash in China' (Nature 456, 545-546; 2008), you incorrectly say that I am professor emeritus, having retired from the College of Life Sciences, Peking University, four years ago. In fact, I retired in February 2006 and do not have emeritus status. Neither did I retain my laboratory there in order for my associate professor to take it over formally as a way of maintaining my influence.

I have kept my laboratory running with the help of a grant from the National Natural Science Foundation of China (NSFC).

When I applied to the NSFC, Peking University guaranteed my lab and equipment until I had completed the work. The associate professor you mention was a co-author on this grant application.

Although I did submit an online posting accusing Yi Rao, the dean of life sciences, of withdrawing the laboratory for use in other applications (http://tinyurl. com/814u9x), I have never proposed that the associate professor should take it over. My aim is that he should be able to use it to continue his research. Keming Cui College of Life Sciences, Peking University, Beijing 100871, People's Republic of China e-mail:ckm@pku.edu.cn 